treatment of postoperative bacterial endophthalmitis. Arch Ophthalmol 1995; 113: 1479-1496.

4 Clark WL, Kaiser PK, Flynn Jr HW, Belfort A, Miller D, Meister DM. Treatment strategies and visual acuity outcomes in chronic postoperative Propionibacterium acnes endophthalmitis. Ophthalmology 1999; 106: 16651670.

5 Aldave AJ, Stein JD, Deramo VA, Shah GK, Fisher DH, Maguire JI. Treatment strategies for postoperative Propionibacterium acnes endophthalmitis. Ophthalmology 1999; 106: 2395-2401.

6 Hall GS, Pratt-Rippin K, Meisler DM, Washington JA, Russel TJ, Miller D. Growth curve for Propionibacterium acnes. Curr Eye Res 1994; 13(6): 465-466.

7 Teichmann KD. Propionibacterium acnes endophthalmitis requiring the intraocular lens removal after failure of medical therapy. J Cataract Refract Surg 2000; 26: 1085-1088.

JA Gonzalez-Martin', P Hiscott ${ }^{2}$ and P Palimar ${ }^{1}$

'Warrington Hospital

Kendrick Wing Lovely Lane

Warrington

Cheshire WA5 1QG, UK

${ }^{2}$ Royal Liverpool University Hospital

Prescot Road

Liverpool, UK

Correspondence: P Palimar

Tel/Fax: +44 1925662395

E-mail: palimar@tinyonline.co.uk

Sir,

\section{Cytarabine-induced corneal toxicity}

Eye (2003) 17, 677-678. doi:10.1038/sj.eye.6700451

Cytarabine (cytosine arabinoside) is a powerful antimetabolite used in the treatment of acute myeloid leukaemia (AML). Corneal toxicity from high-dose intravenous cytarabine therapy has been described in the literature ${ }^{1}$ and routine prophylaxis with topical steroids is now an established part of the treatment protocol. ${ }^{2}$ Only two cases have been reported of corneal toxicity occurring at low doses. One case occurred with an intermittent subcutaneous regimen. ${ }^{3}$ A second case was reported with a continuous intravenous regimen. ${ }^{4}$ The cytotoxic activity of cytarabine is related to the concentration and duration of exposure, both of which vary with different modes of administration. There are no reports of corneal toxicity in patients receiving an intermittent, intravenous low-dose regimen, and currently no recommendations have been made for prophylaxis in these cases.

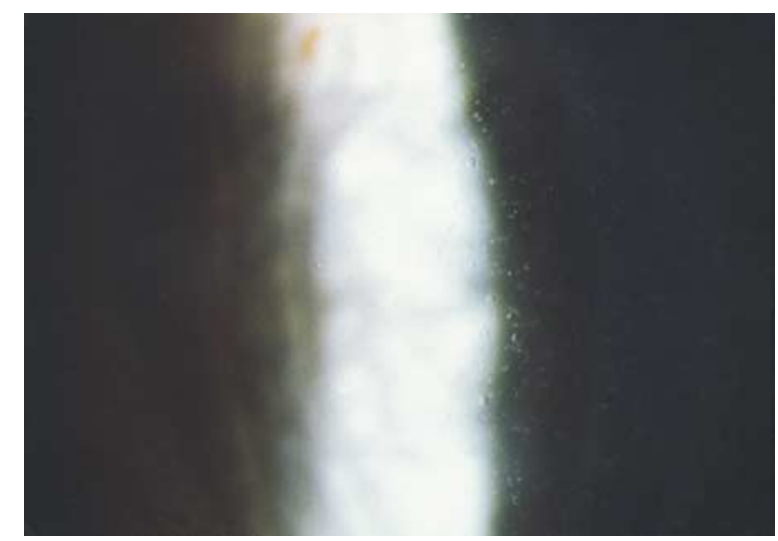

Figure 1 Corneal epithelial microcysts visible with direct slit-beam illumination.

\section{Case report}

A 39-year-old male presented with a 1-day history of blurred vision, severe discomfort and photophobia following a 10-day course of intermittent low-dose intravenous cytarabine therapy for AML. He had received $200 \mathrm{mg} / \mathrm{m}^{2}$ every $12 \mathrm{~h}$ for 9 days prior to the onset of symptoms and had not been using topical steroid prophylaxis. On examination he had an uncorrected visual acuity of $6 / 9$ in each eye. Severe blepharospasm and moderate conjunctival inflammation were present. Bilateral corneal epithelial microcysts, more densely distributed in the centre of the cornea than in the midperiphery were noted, with a clear zone of about $1.5 \mathrm{~mm}$ in the corneal periphery (Figure 1). The anterior chamber in both eyes was free of inflammation and the intraocular pressure was normal. Treatment was commenced with G. dexamethasone $0.1 \% 2$ hourly and the symptoms resolved completely within 3 days. The microcysts disappeared after 7 days and the steroid drops were subsequently tapered and then stopped over the following week.

\section{Comment}

Cytarabine corneal toxicity has mainly been associated with high-dose intravenous therapy $\left(>1 \mathrm{~g} / \mathrm{m}^{2}\right)$. This drug is known to penetrate the blood-brain barrier after intravenous infusion and is also found in the aqueous and tears. Typically, corneal toxicity occurs after 5-7 days of treatment and can be prevented by using topical corticosteroids. ${ }^{1,2}$ In this patient, because of the relatively low-dose regimen, prophylactic steroid therapy was not used and typical corneal toxicity occurred as a consequence.

The mechanism of cytarabine-induced microcyst formation is not currently known. Rapid cycling cells are most sensitive to the actions of cytarabine. Corneal 
epithelial stem cells have a long cell cycle time and consequently are unlikely to be vulnerable to its effects. They give rise to more differentiated transient amplifying cells (TACs) located within the basal cell layer, which divide more frequently and therefore might be expected to be more vulnerable. In the patient studied here there was a peripheral clear zone in the corneal epithelium, suggesting that the central epithelial cells were relatively more sensitive than the peripheral TACs. These 'transitional' peripheral epithelial cells appear to be a population of very early TACs, which display some of the characteristics of stem cells. ${ }^{5}$ A functional difference in behaviour between this peripheral zone of epithelium and the central zone is suggested clinically by findings in the $\beta$ ig-h 3 corneal dystrophies (eg Granular, Lattice, Avelino and Honeycomb dystrophies).

Characteristically, the peripheral epithelium is clear in these disorders.

The mechanism by which a topical corticosteroid reduces this toxic effect is unclear. As cytarabine inhibits DNA synthesis at the level of the DNA polymerase, a reduction in DNA replication by the steroid would make the corneal epithelial cells less susceptible to the effects of this drug. ${ }^{2}$

Although uncommon, this case confirms that cytarabine corneal toxicity can occur with low-dose regimens and it is recommended that all patients receiving cytarabine therapy should receive prophylactic topical steroids.

\section{References}

1 Hopen G, Mondino BJ, Johnson BL, Chevernick PA. Corneal toxicity with systemic cytarabine. Am J Ophthalmol 1981; 91: 500-504.

2 Lass JH, Lazarus HM, Reed MD, Herzig RH. Topical corticosteroid therapy for corneal toxicity from systemically administered cytarabine. Am J Ophthalmol 1982; 94: 617-621.

3 Kumar L, Dua H, Agarwal S, Singh S, Kochupillai V. Ocular toxicity of low dose cytosar. New Zealand Med J 1987; 100: 361.

4 Barletta J, Fanous M, Margo C. Corneal and conjunctival toxicity with low-dose cytosine arabinoside. Am J Ophthalmol 1992; 113: 587-588.

5 Lauweryns B, van den Oord JJ, Missotten L. The transitional zone between limbus and peripheral cornea. An immunohistochemical study. Invest Ophthalmol Vis Sci 1993; 34: 1991-1999.

\section{J Lochhead, JF Salmon and AJ Bron}

The Oxford Eye Hospital

Radcliffe Infirmary Woodstock Road

Oxford OX2 6HE, UK

Correspondence: Dr J Lochhead

Tel: +4401865224360

Fax: +4401865 224515

E-mail: jonlochh@AOL.com 